\title{
Tipologías de estilos de vida en jóvenes universitarios
}

\author{
Lifestyles typologies in college youth
}

María Teresa Varela-Arévalo', Andrés Felipe Ochoa-Muñoz², José Rafael Tovar-Cuevas³

\begin{abstract}
1 Psicóloga, MsC en Epidemiología. Profesora, Departamento de Ciencias Sociales, Grupo de investigación Salud y Calidad de Vida. Pontificia Universidad Javeriana. Cali, Colombia. e-mail: mtvarela@javerianacali.edu.co

2 Estadístico, Estudiante de Maestría en Estadística. Joven investigador Colciencias, Grupo de investigación Salud y Calidad de Vida. Pontificia Universidad Javeriana. Cali, Colombia. e-mail: andresochoa7788@gmail.com

3 Estadístico, PhD en Estadística. Profesor asistente, Escuela de Estadística, Universidad del Valle. Cali, Colombia. e-mail: jose.r.tovar@correounivalle.edu.co
\end{abstract}

\begin{abstract}
Resumen
Introducción: La evidencia actual sobre la relación entre los estilos de vida y las principales problemáticas de salud mundial es amplia, dando cuenta del aumento en las cifras de comportamientos de riesgo y de sus impactos en la salud de la población. Objetivo: Identificar agrupaciones entre los jóvenes matriculados en una universidad privada de Cali, a partir de ocho dimensiones relacionadas con sus estilos de vida. Materiales y métodos: Se aplicó el Cuestionario de Estilos de Vida de Jóvenes Universitarios, que permitió clasificar las prácticas de los jóvenes en saludables y no saludables a 370 estudiantes con edades entre 18 y 25 años. Se utilizaron modelos de regresión para respuesta binaria y una combinación de métodos factoriales con análisis de conglomerados para obtener tipificación de estilos de vida. Se trabajó con ayuda de los paquetes Stats para los modelos de regresión y FactoClass para el análisis multivariado con el software R. Resultados: Fue posible establecer tres conformaciones tipológicas: 1) hombres sin pareja, que no trabajan, con prácticas no saludables en actividad física; 2) mujeres sin pareja, que no trabajan, con prácticas no saludables de actividad física y alto nivel de estrés; 3) mujeres sin pareja, que no trabajan, con prácticas no saludables de actividad física. Conclusiones: Las tipologías encontradas permitirán a la universidad realizar intervenciones focalizadas que consideren las características propias de diferentes tipos de jóvenes, logrando mejores resultados y optimizando los recursos disponibles.
\end{abstract}

Palabras clave: Conductas saludables; adultos jóvenes; análisis de regresión; género y salud; salud del adolescente. (Fuente: DeCS, Bireme).

\begin{abstract}
Introduction: The current evidence on the relationship between lifestyles and the main health problems in the world is wide, taking into account the increase in the numbers of behaviors of risk and their impacts on the health of the population Objetive: The aim of the study was to describe life styles of private college youth from Cali, according to their practices in eight dimensions, and to identify clustering of them. Materials and methods: The study sample consisted of 370 youth with ages between 18 and 25 years, who answered the Lifestyle Questionnaire for University Students - CEVJU-R2, which classifies healthy and unhealthy practices. Regression models for binary response factor and a combination of cluster analysis methods were used. The stats packages for the regression models and FactoClass
\end{abstract}


(10) for multivariate analysis with the R software were used. Results: With binary regression models and combination of multivariate methods, it was possible to find three clusters: 1) men, without couple, not working, with unhealthy physical activity practices; 2 ) women, without couple, not working, with unhealthy physical activity practices and high level of stress; and 3) women, without couple, not working, with unhealthy physical activity practices. Conclusions: Typologies found will allow the university to make targeted interventions that consider the characteristics of different types of youth, achieving better results and optimizing the available resources.

Keywords: Health behavior; young adult; regression analysis; gender and health; adolescent health. (Source: DeCS, Bireme).

\section{Introducción}

La evidencia actual sobre la relación entre los estilos de vida y las principales problemáticas de salud mundial es amplia, dando cuenta del aumento en las cifras de comportamientos de riesgo y de sus impactos en la salud de la población. Los estilos de vida abarcan un conjunto amplio de hábitos en aspectos como la alimentación, la sexualidad, el tiempo de ocio, la actividad física, el consumo de sustancias psicoactivas, el seguimiento de recomendaciones médicas, el manejo del estrés psicosocial, etc ${ }^{1,2}$.

La juventud ha sido tradicionalmente considerada como una etapa de mayor riesgo, en la que aumenta la incidencia de embarazos precoces, abortos, infecciones de transmisión sexual (ITS) y VIH/sida, lesiones y muertes por accidentes de tránsito, consumo de sustancias psicoactivas, trastornos de la conducta alimentaria, entre otros, dada la búsqueda de identidad, autosuficiencia e independencia, propia de la transición de la adolescencia a la adultez ${ }^{3,4}$. Asimismo, debido a las ocupaciones de los jóvenes, el ingreso a la universidad, el menor control parental, las actividades sociales y el uso frecuente de dispositivos tecnológicos, disminuye su nivel de actividad física, sus hábitos de alimentación cambian y los niveles de estrés aumentan ${ }^{5,6}$. Todo lo anterior configura estilos de vida particulares en esta población, tendiendo a ser considerados de alto riesgo ${ }^{7}$.

Los estudios que buscan describir los estilos de vida de jóvenes, principalmente de estudiantes universitarios, son abundantes incluso en Colombia, pero se han enfocado principalmente en evaluar comportamientos no saludables de manera aislada, si bien la evidencia señala cada vez con mayor fuerza que los hábitos de salud coexisten y se agrupan entre sí1,2,6,8-14.

De acuerdo con esto, son pocos los intentos por identificar tipologías o "clúster" de hábitos del estilo de vida de esta población, lo que resultaría muy útil para establecer patrones y grupos con mayor riesgo, y por tanto, focalizar las intervenciones y ajustarlas a grupos con características específicas en las instituciones educativas ${ }^{15}$.

Los pocos estudios disponibles que han perseguido este objetivo, se han centrado en poblaciones generales de adultos, niños o trabajadores ${ }^{16-21}$. No obstante, recientemente se han llevado a cabo estudios en universitarios, los cuales han identificado la clara existencia de "tipos de estudiantes" en relación con sus hábitos de salud, siendo más frecuentes las agrupaciones de hábitos no saludables que conforman perfiles de alto riesgo para estos jóvenes.

Un estudio con 1.262 estudiantes de primer año de una universidad en Alemania evaluó la prevalencia de comportamientos de riesgo en salud (consumo de frutas y verduras, ejercicio, tabaquismo y consumo excesivo de alcohol), su agrupación y la intención de cambio de comportamiento. Al respecto encontraron una alta prevalencia de los cuatro comportamientos en salud evaluados, sólo el $2 \%$ de los jóvenes no tenía ningún comportamiento de riesgo, el 10\% presentaba un sólo comportamiento de riesgo, el $35 \%$ presentaba dos, el 35\% tenía tres y el $18 \%$ cuatro comportamientos de riesgo, igualmente encontraron una baja intención de cambio de comportamiento ${ }^{7}$. 
Otro estudio en Alemania de tipo longitudinal con 556 adolescentes y jóvenes alemanes evaluó la asociación en el tiempo entre patrones de comportamientos en salud, peso y estado de salud autopercibida, encontrado cuatro clúster. De estos, el relacionado con baja actividad física, alto consumo de entretenimiento digital y dieta de baja calidad tuvo la mayor influencia en el sobrepeso. Por su parte, el clúster relacionado con un menor incremento de peso se caracterizó por un alto nivel de actividad física, un consumo moderado de entretenimiento digital y una dieta de alta calidad ${ }^{15}$.

Un estudio con 732 adultos jóvenes de una universidad en Qatar exploró la agrupación de hábitos de alimentación, índice de masa corporal (IMC) y actividad física, identificó tres clúster. El primero se caracterizó por factores de alto riesgo, incluyendo prácticas de alimentación poco saludables, baja actividad física y alto IMC; el segundo estuvo compuesto por factores de riesgo moderados como hábitos alimentarios medianamente saludables, realización de actividad física, bajo consumo de televisión y computadores y bajo IMC; mientras que el tercer clúster se caracterizó por un bajo riesgo con hábitos alimentarios saludables y realización de actividad física moderada ${ }^{6}$.

Similar al anterior, un estudio con 410 estudiantes universitarios del Reino Unido evaluó la prevalencia y agrupación de comportamientos no saludables, entre ellos el estrés psicosocial, la actividad física, el consumo de frutas y verduras, el consumo excesivo de alcohol y el tabaquismo. Además de encontrar una alta prevalencia de hábitos no saludables, el estudio reportó la conformación de tres clúster: el primero denominado no saludable o de riesgo, caracterizado por alto estrés psicosocial, baja actividad física, bajo consumo de frutas y verduras y consumo ocasional o regular de tabaco; el segundo denominado de riesgo moderado, conformado por niveles moderados de estrés psicosocial, actividad física y consumo de frutas y verduras, pero con un alto consumo de alcohol y una alta proporción de fumadores; y el tercero denominado saludable o de bajo riesgo, caracterizado por bajo estrés psicosocial, alta actividad física, consumo de más de cinco porciones de frutas y verduras, consumo moderado de alcohol y una alta proporción de no fumadores ${ }^{14}$.

Por su parte, un estudio con 1.463 estudiantes universitarias mujeres de Estados Unidos evaluó la prevalencia y agrupación de comportamientos de mantenimiento de la salud (consumo de frutas y verduras, actividad física y tamizaje de cáncer cervical) y comportamientos de alto riesgo (consumo riesgoso de alcohol, tabaquismo y no uso de medidas de protección durante las relaciones sexuales). Los resultados mostraron que el $65 \%$ de las mujeres presentaban dos o más comportamientos no saludables y señalaron la presencia de tres clúster: el primero constituido por mujeres con bajo mantenimiento de la salud y altos comportamientos de riesgo; en segundo con mujeres con bajo mantenimiento de la salud y bajos comportamientos de riesgo; y el tercero con mujeres con alto mantenimiento de la salud y comportamientos moderados de riesgo ${ }^{13}$.

Si se tiene en cuenta que el periodo transicional en el que se encuentran los jóvenes universitarios es crítico para el desarrollo de hábitos de salud que tenderán a permanecer hasta la adultez, resulta importante en esta etapa favorecer actitudes y prácticas positivas para mantener la salud y para evitar los precursores biológicos del desarrollo de enfermedades crónicas en esa etapa ${ }^{6}$. Además, dado que pasan gran parte de su tiempo en la universidad, ésta se convierte en un espacio privilegiado en el que se pueden promover hábitos, entornos físicos y sociales que promuevan la salud y la calidad de vida de sus estudiantes. De acuerdo con lo anterior, este estudio se propuso describir los hábitos de actividad física, tiempo de ocio, alimentación, consumo de sustancias psicoactivas, sueño, afrontamiento, sexualidad y estrés en jóvenes de una universidad de Cali-Colombia y establecer tipologías de estilos de vida. Estos aspectos fueron evaluados con el Cuestionario de Estilos de Vida de Jóvenes Universitarios (CEVJU-R), el cual es una medida de autoinforme validada para universitarios colombianos y ampliamente utilizada en el país y en Iberoamérica². 


\section{Materiales y métodos}

Participantes: Se realizó un estudio transversal de corte descriptivo con una muestra de 370 estudiantes de una universidad privada de la ciudad de Cali-Colombia. Los participantes debían cumplir como criterios de inclusión ser estudiantes activos al momento de la medición, mayores de edad y manifestar intención de participar voluntariamente en el estudio firmando un consentimiento informado. La elección de la muestra se realizó de manera intencional, conservando la representatividad por facultad a la que pertenecían los estudiantes y la ubicación semestral.

Instrumento: Se utilizó el Cuestionario de Estilos de Vida en Jóvenes Universitarios² ${ }^{2}$ en su versión CEVJU-R2, el cual está compuesto por un apartado de datos sociodemográficos y un conjunto de 63 ítems que indagan información sobre ocho dimensiones de los estilos de vida: actividad física, tiempo de ocio, alimentación, consumo de alcohol, cigarrillo y drogas ilegales, sueño, afrontamiento, sexualidad y estrés. El CEVJU-R2 evalúa las prácticas en cada dimensión del estilo de vida (opciones de respuesta con escalamiento tipo Likert), además de variables relacionadas con éstas dimensiones, entre ellas las motivaciones y los recursos percibidos. Los resultados presentados en este artículo corresponden a los puntajes obtenidos para las prácticas en las dimensiones, en las cuales se puntúa asignando un valor entre uno y cuatro a la opción de respuesta del evaluado. La suma de valores del individuo es comparada con el valor medio del intervalo formado por las posibles puntuaciones, de modo que si la puntuación obtenida es superior al punto medio, se pueden clasificar las prácticas del sujeto como no saludables. (Tabla 1)

Tabla 1. Puntuaciones posibles de los estilos de vida de jóvenes universitarios

\begin{tabular}{lrrr}
\hline \multicolumn{1}{c}{ Dimensión } & Ítems & $\begin{array}{c}\text { Puntuaciones } \\
\text { posibles }\end{array}$ & $\begin{array}{c}\text { Punto } \\
\text { medio }\end{array}$ \\
\hline Actividad física & $1-3$ & 3 a 12 & 7,5 \\
Tiempo de ocio & $8-10$ & 3 a 12 & 7,5 \\
$\begin{array}{l}\text { Alimentación } \\
\begin{array}{l}\text { Consumo de alcohol, } \\
\text { cigarrillo y drogas }\end{array}\end{array}$ & $15-21$ & 7 a 28 & 17,5 \\
ilegales & $26-30$ & 5 a 23 & 14 \\
$\begin{array}{l}\text { Sueño } \\
\text { Afrontamiento }\end{array}$ & $35-37$ & 3 a 12 & 7,5 \\
Sexualidad & $42-46$ & 5 a 20 & 12,5 \\
Estrés & $53-59$ & 6 a 24 & 15 \\
\hline
\end{tabular}

Después de obtener las frecuencias de las respuestas de los estudiantes a cada ítem, se observó que algunos estudiantes no contestaron todos los ítems de la dimensión, por ello se decidió re-escalar las respuestas para no perder un número importante de sujetos. Para esto se utilizó como criterio puntuar de nuevo aquellos individuos que habían respondido al $80 \%$ o más de los ítems de la respectiva dimensión, aplicándole una nueva escala de calificación proporcional a la original. En la tabla 2 aparecen las dimensiones y los valores de los puntajes después de hacer el re-escalamiento.

Tabla 2. Recalificación de la escala según jóvenes que responden por lo menos el $80 \%$ de los ítems en la dimensión

\begin{tabular}{|c|c|c|c|c|}
\hline Dimensión & $\begin{array}{c}\text { Número } \mathrm{X} \text { de ítems que } \\
\text { responde }\end{array}$ & $\begin{array}{c}\text { Puntuaciones posibles } \\
\text { (rango) }\end{array}$ & $\begin{array}{l}\text { Punto medio } \\
(\text { Pm) }\end{array}$ & $\begin{array}{l}\text { Número de estudiantes } \\
\text { recalificados }\end{array}$ \\
\hline Alimentación & $>=6$ & $\begin{array}{l}\text { Si } X=6 \ldots[6-24] \\
\text { Si } X=7 \quad \ldots[7-28]\end{array}$ & $\begin{array}{r}\text { Si } X=6 \ldots \text { Pm=15 } \\
\text { Si X=7 ... } \\
\text { Pm=17.5 }\end{array}$ & 9 \\
\hline $\begin{array}{l}\text { Consumo de alcohol, cigarrillo y } \\
\text { drogas ilegales }\end{array}$ & $>=4$ & $\begin{array}{l}\text { Si } X=4 \ldots[4-19] \\
\text { Si } X=5 \ldots[5-23]\end{array}$ & $\begin{array}{r}\text { Si X=4 } \\
\ldots . P m=11.25 \\
\text { Si } X=5 \ldots P m=14\end{array}$ & 15 \\
\hline Afrontamiento & $>=4$ & $\begin{array}{l}\text { Si X }=4 \ldots[4-16] \\
\text { Si X } X=5 \ldots[5-20]\end{array}$ & $\begin{array}{r}\text { Si } X=4 \ldots . P m=10 \\
\text { Si } X=5 \ldots \\
P m=12.5\end{array}$ & 9 \\
\hline Sexualidad & $>=5$ & $\begin{array}{l}\text { Si } X=5 \ldots[5-20] \\
\text { Si } X=6 \ldots[6-24]\end{array}$ & $\begin{array}{r}\text { Si } X=5 \ldots \\
P m=12.5 \\
\text { Si } X=6 \ldots P m=15\end{array}$ & 4 \\
\hline Estrés & $>=5$ & $\begin{array}{l}\text { Si } X=5 \ldots[5-25] \\
\text { Si } X=6 \ldots[6-30]\end{array}$ & $\begin{array}{l}\text { Si } X=5 \ldots P m=15 \\
\text { Si } X=6 \ldots P m=18\end{array}$ & 2 \\
\hline
\end{tabular}


Análisis de datos: El análisis estadístico de los datos fue realizado con un archivo en el que se incluyeron los registros de todos los individuos que contestaron la totalidad de los ítems del cuestionario, de modo que la muestra final de estudio estuvo compuesta por 228 registros de igual número de estudiantes. La construcción de tipologías o grupos de universitarios con ciertas características asociadas a sus estilos de vida, partió de una selección de variables de caracterización, para lo que se emplearon métodos estadísticos que parten de dos perspectivas diferentes:

\section{Selección de variables usando modelos de} regresión con respuesta binaria. En este procedimiento se realizó la selección de variables, tomando como variable respuesta la clasificación del estudiante en cada dimensión, después de comparar la puntuación obtenida por sus respuestas con el valor medio del intervalo de puntajes en la misma. Se tomaron como covariables el género, la edad, el semestre, estar trabajando y tener pareja actualmente.

Se evaluaron siete modelos de regresión con respuesta binaria, con el objetivo de establecer las covariables que modifican la probabilidad de que un estudiante sea clasificado como no saludable (evento éxito) respecto a sus prácticas relacionadas con cada una de las dimensiones de estilos de vida. En las dimensiones para las que el número de individuos con prácticas no saludables era próxima a la mitad de los individuos, se utilizó un modelo de regresión logística (enlace logit) mientras que, cuando se tenía que más del $80 \%$ de los jóvenes había sido clasificado en alguna de las dos categorías, se ajustó un modelo log-log complementario para hacer la selección de las covariables $^{22-24}$.

Para establecer las variables con mayor peso sobre la probabilidad de tener prácticas no saludables, se realizaron pruebas de hipótesis sobre los parámetros del modelo y se utilizó un procedimiento de selección hacia atrás (backward), asumiendo que si la significancia estadística asociada a la presencia de la covariable en el modelo, era menor o igual a 0.1 , la misma podía ser seleccionada para construir la tipología.
2. Combinación de métodos factoriales y de análisis de conglomerados para establecer las tipologías. Con este procedimiento se buscó establecer grupos de estudiantes con características en común. Se utilizó el Análisis de Correspondencias Múltiples (ACM) con las variables que hacen referencia a la clasificación de práctica saludable y las variables usadas como covariables en el procedimiento anterior. El ACM permite obtener un conjunto de pesos asociados a las variables seleccionadas, en forma de coordenadas de ejes dentro de un plano cartesiano. Dichas coordenadas, permiten establecer agrupaciones de sujetos (clusters) utilizando un algoritmo mixto de clasificación desarrollado por Lebart25.

El análisis descriptivo de los datos fue realizado con la ayuda del software estadístico SPSS versión 21. Para obtener las tipologías, se utilizó el software libre $\mathrm{R}$ en su versión 3.1.2. Las agrupaciones mediante clusters fueron obtenidas con la ayuda de la librería FactoClass instalada en el $\mathrm{R}^{26}$. El análisis bivariado de los datos incluyó la realización de pruebas chi cuadrado para evaluar asociación entre variables y pruebas exactas de Fisher en los casos en los que el número esperado de casos por celda era menor de cinco. Se utilizó un nivel de confianza del $90 \%$ para realizar los análisis previos a la construcción de las tipologías.

\section{Resultados}

Entre los 370 registros del archivo de datos, se escogieron $228(62 \%)$ individuos que contaban con la información completa de todas las variables medidas en el estudio. Entre los 228 seleccionados, el $52.4 \%$ pertenecía al sexo femenino. La edad promedio fue 20 años (rango entre 18 y 25 años). El $60.1 \%$ de los estudiantes tenían edades entre 18 y 20 años, el $20.1 \%$ tienen edades comprendidas entre los 21 y 22 años y los demás pertenecían al grupo de edades entre 23 y $\operatorname{los} 25$ años.

En cuanto a la ubicación semestral, el 38.6\% de los individuos adelantaba entre primero y tercer semestre de la carrera, el $38.6 \%$ estaba entre cuarto y séptimo semestre y los demás cursaban 
octavo o un semestre superior. El $44.9 \%$ de los jóvenes afirmó tener pareja estable y un $22 \%$ de los jóvenes se encontraba trabajando al momento de realizar el estudio. Los resultados de los análisis bivariados usando tablas de contingencia aparecen en la tabla 3. La dimensiones de actividad física, consumo de sustancias psicoactivas - SPA (alcohol, cigarrillo y drogas ilegales) y estrés, tienen asociación con la variable sexo.

Tabla 3. Valores p obtenidos para rechazar la hipótesis de independencia entre pares de variables seleccionadas para el estudio

\begin{tabular}{lrrrrr}
\hline $\begin{array}{c}\text { Dimensión vs } \\
\text { variables } \\
\text { sociodemográficas }\end{array}$ & Sexo & Edad & Semestre & Trabajar & Pareja \\
\hline Actividad física & $.000^{*}$ &. $\mathbf{4 3 5}$ & .519 & $.082^{*}$ & .912 \\
Tiempo de ocio & .214 & $\mathbf{. 5 6 3}$ & .747 & $.071^{*}$ & .564 \\
Alimentación & .610 &. $\mathbf{4 4 4}$ &. $\mathbf{3 0 6}$ & .333 & .798 \\
Consumo de SPA & $.003^{*}$ & $\mathbf{. 3 3 3}$ &. $\mathbf{4 6 1}$ & $\mathbf{. 3 7 9}$ & .205 \\
Sueño & .237 &. $\mathbf{2 3 1}$ & .734 & .838 & .863 \\
Afrontamiento & .163 & $\mathbf{1}$ & .239 & .811 & .408 \\
Estrés & $.000^{*}$ & $\mathbf{. 3 7 4}$ & .177 & .595 & .319 \\
\hline En negrilla los valores p obtenidos usando la prueba exacta de Fisher
\end{tabular}

Más del $60 \%$ de los jóvenes quedó clasificado dentro del grupo con prácticas saludables en todas las dimensiones, excepto en la dimensión actividad física, donde la mayoría de los jóvenes tiene prácticas no saludables. Las dimensiones con mayor número de participantes clasificados (más de $85 \%$ ) con prácticas saludables fueron alimentación, consumo de SPA, sueño, afrontamiento y sexualidad. (Tabla 4)

Al analizar los indicadores de cada dimensión del estilo de vida, se observó que, en actividad física, sólo el 38\% utiliza otro medio de transporte como caminar o montar bicicleta para desplazarse a un lugar cercano y el $23 \%$ practica algún deporte con fines competitivos.

En la dimensión tiempo de ocio se observa que cerca del $60 \%$ de los jóvenes realiza frecuentemente actividades para descansar y relajarse, comparten con la familia, amigos o pareja y realizan frecuentemente actividades culturales o artísticas. En cuanto a la alimentación una gran proporción de jóvenes reportan consumir con frecuencia comidas rápidas, fritos, mecatos o dulces, gaseosas o bebidas artificiales; no obstante afirman desayunar, almorzar y comer en horarios habituales. En la dimensión consumo de SPA, la mayoría se consideran consumidores sociales de alcohol, pocos son fumadores habituales, y solo un $4 \%$ reporta consumir drogas ilegales con frecuencia.

Tabla 4. Distribución de las dimensiones de los estilos de vida de los estudiantes universitarios

\begin{tabular}{|c|c|c|c|c|c|c|}
\hline Dimensión & Categoría & $\mathbf{N}$ & $\%$ & Variable seleccionada & OR & p-value $>10 \%$ \\
\hline \multirow{2}{*}{ Actividad física } & Saludable & 129 & 35.2 & \multirow{2}{*}{ Sexo (mujer) } & \multirow{2}{*}{3.518} & \multirow{2}{*}{0.000} \\
\hline & No saludable & 237 & 64.8 & & & \\
\hline \multirow{2}{*}{ Tiempo de ocio } & Saludable & 235 & 67 & \multirow{2}{*}{ No trabajar actualmente } & \multirow{2}{*}{0.622} & \multirow{2}{*}{0.086} \\
\hline & No saludable & 116 & 33 & & & \\
\hline \multirow{2}{*}{ Alimentación } & Saludable & 347 & 95.3 & \multirow{2}{*}{ - } & \multirow{2}{*}{-} & \multirow{4}{*}{0.012} \\
\hline & No saludable & 17 & 4.7 & & & \\
\hline \multirow{2}{*}{ Consumo de SPA } & Saludable & 258 & 93.1 & \multirow{2}{*}{ Sexo (mujer) } & \multirow{2}{*}{0.201} & \\
\hline & No saludable & 19 & 6.9 & & & \\
\hline \multirow{2}{*}{ Sueño } & Saludable & 323 & 89.2 & \multirow{2}{*}{-} & \multirow{2}{*}{-} & - \\
\hline & No saludable & 39 & 10.8 & & & \\
\hline \multirow{2}{*}{ Afrontamiento } & Saludable & 336 & 92.6 & \multirow{2}{*}{-} & \multirow{2}{*}{-} & \\
\hline & No saludable & 27 & 7.4 & & & \\
\hline \multirow{2}{*}{ Sexualidad } & Saludable & 282 & 100 & \multirow{2}{*}{ - } & \multirow{2}{*}{-} & \\
\hline & No saludable & 0 & 0 & & & \\
\hline \multirow{2}{*}{ Estrés } & Saludable & 232 & 63.6 & \multirow{2}{*}{ Sexo (mujer) } & \multirow{2}{*}{2.811} & \multirow{2}{*}{0.000} \\
\hline & No saludable & 133 & 36.4 & & & \\
\hline
\end{tabular}

Nota: Datos obtenidos mediante la regresión logística como método clasificador 
Para la dimensión asociada al sueño se encontró que el $43,4 \%$ de los jóvenes afirma que frecuentemente, no se acuestan ni se levantan a horas habituales, y una pequeña pero importante proporción de jóvenes reporta despertarse de noche y tener dificultades para seguir durmiendo. En cuanto al afrontamiento, se encontró que la mayoría tienen buenas estrategias para hacerle frente a las situaciones difíciles que se les presentan, como analizar en qué consiste la situación, evaluar si pueden hacer algo para cambiarla, buscar apoyo por parte de otras personas y aceptar aquellas situaciones que no pueden cambiar.

En cuanto a las prácticas relacionadas con la sexualidad, se encontró que de los jóvenes que han tenido relaciones sexuales el último año, solo el $36 \%$ utiliza preservativos en todas las relaciones sexuales y el 37\% utiliza algún método anticonceptivo. El 66\% nunca se ha realizado una prueba de detección de ITS. En la dimensión de estrés se encontró que el $44 \%$ de los jóvenes, frecuentemente se han sentidos estresados y preocupados y se sienten enojados por cosas que están fuera de su control. Cerca del $20 \%$ de los jóvenes casi siempre sienten que se les acumulan las dificultades y no pueden manejarlas y se sienten inseguros para enfrentar sus problemas personales.

\section{Tipologías de estilos de vida}

Usando modelos de regresión, se observó que las mujeres tienen mayor probabilidad de tener prácticas no saludables en las dimensiones actividad física y estrés; así mismo, no estar trabajando actualmente modifica de manera importante la probabilidad de tener prácticas no saludables en la dimensión tiempo de ocio. También se observó en la dimensión consumo de alcohol, cigarrillo y drogas ilegales, que las mujeres tienen menor probabilidad de tener practicas no saludables. Para las dimensiones alimentación, sueño, afrontamiento y sexualidad no se identificaron covariables cuya importancia estadística permitiera seleccionarlas como posibles factores que modifican la probabilidad de encontrar prácticas no saludables en estas dimensiones.
A partir de lo anterior, se establecieron entonces las siguientes tipologías de estudiantes:

1. Mujeres con prácticas no saludables respecto a la actividad física.

2. Jóvenes que trabajan con prácticas no saludables en tiempo de ocio.

3. Mujeres con prácticas saludables en consumo de alcohol, cigarrillo y drogas ilegales.

4. Mujeres con prácticas no saludables en el manejo del estrés.

Por otro lado, mediante el uso de métodos multivariados se obtuvieron otras tipologías. Para encontrar grupos de jóvenes con características asociadas a sus estilos de vida, se utilizó la propuesta metodológica desarrollada por Lebart 25 , en la cual se combinan el método factorial y el análisis de conglomerados. El procedimiento se aplicó a las variables género, edad, semestre, tener pareja, trabajar actualmente y la variable de clasificación de prácticas saludables en cada dimensión; excepto la dimensión sexualidad, pues todos los jóvenes presentaron practicas saludables. Todas las variables se trabajaron en su forma cualitativa y se eliminaron los datos faltantes por filas, puesto que era necesario tener la información completa. Por tanto, los grupos o conglomerados encontrados dependieron de un Análisis de Correspondencia Múltiple (ACM) realizado sobre 228 registros con datos completos en cada una de las variables consideradas. Se obtuvieron tres conglomerados cada uno con características específicas, que permitió establecer las siguientes tipologías de comportamientos no saludables:

1. Hombres cuyas edades oscilan entre 18 a 20 años, cursando semestres de primero a séptimo, que no tienen pareja y no trabajan, con prácticas no saludables en actividad física.

2. Mujeres cuyas edades oscilan entre 18 a 22 años, cursando semestres de cuarto a séptimo, que no tienen pareja y no trabajan, con prácticas no saludables de actividad física y estrés.

3. Mujeres cuyas edades oscilan entre 21 a 25 años, cursando semestres entre octavo y decimo, que no tienen pareja y no trabajan, con prácticas no saludables de actividad física. 
Las tipologías encontradas por el análisis multivariado se realizan teniendo en cuenta las frecuencias observadas, de manera que se explican las características de cada tipología en función de lo más frecuente. En el gráfico 1, se observan los tres clúster de jóvenes ubicados en el plano factorial.

\section{Gráfico 1. Identificación de tipologías de jóvenes universitarios según sus estilos de vida}

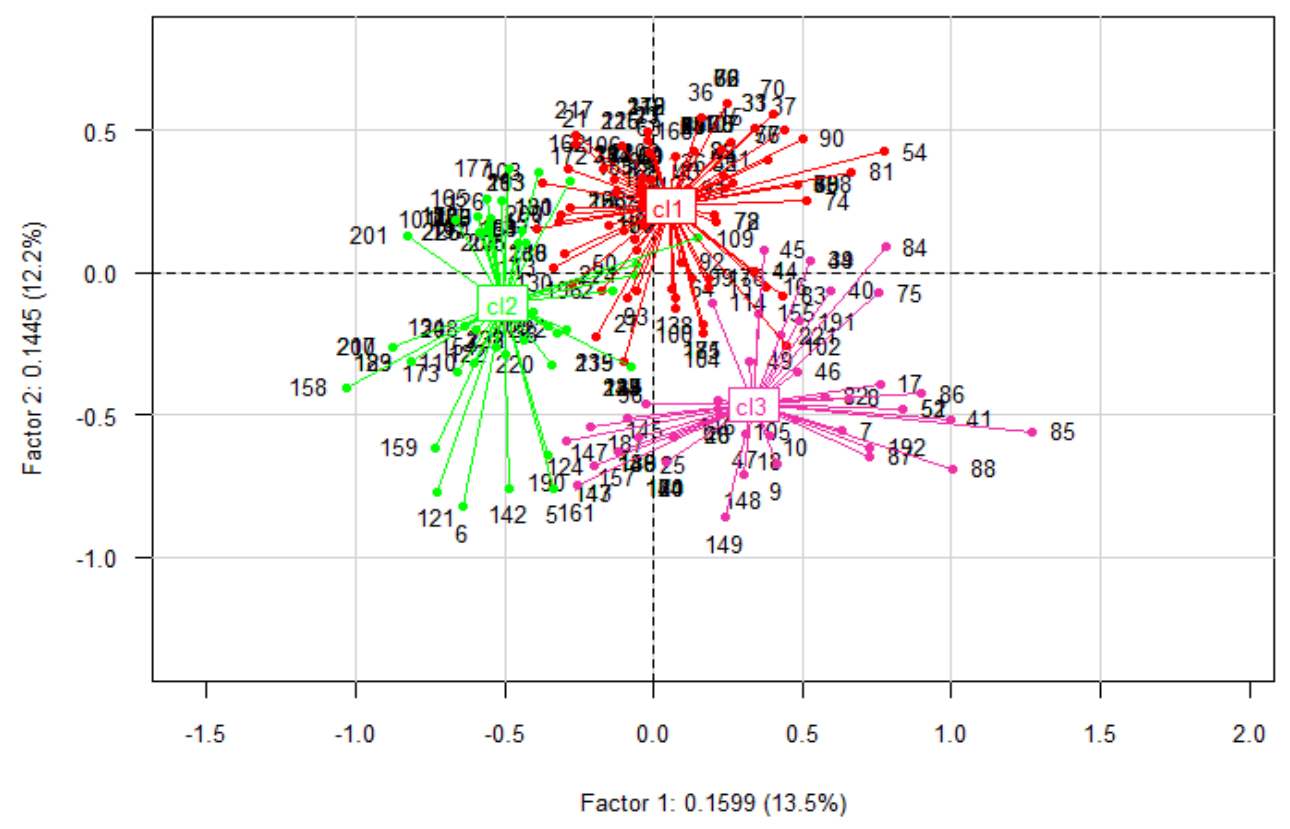

\section{Discusión}

Los propósitos de este estudio fueron, por un lado, describir los hábitos del estilo de vida de jóvenes universitarios $y$, adicionalmente establecer si dichos hábitos se agrupan en tipologías. Los resultados muestran que, en general, las prácticas menos saludables se presentaron en la dimensión de actividad física, reflejando un alto porcentaje de jóvenes sedentarios que no cumplen con las recomendaciones mínimas de actividad física.

$\mathrm{Si}$ bien algunos estudios anteriores sobre ese aspecto en la institución donde se llevó a cabo el estudio, habían señalado el problema del sedentarismo como la principal problemática del estilo de vida a intervenir, cabe señalar que la proporción de estudiantes con bajo nivel de actividad física ha disminuido de $73 \%$ en el 20091 a $70,5 \%$ en el $2011^{2}$ y a $65 \%$, actualmente.
La disminución en los porcentajes de jóvenes sedentarios podría deberse a un aumento en el tiempo que los jóvenes caminan para desplazarse a lugares cercanos, posiblemente fomentado por los cambios en el sistema de transporte de la ciudad, que exige a los usuarios caminar hasta los paraderos establecidos que pueden estar en cierta medida alejados de sus destinos finales.

Adicionalmente, podría pensarse en la influencia de la cultura fitness que actualmente ha puesto en auge la realización de ejercicio como parte del estilo de vida, principalmente entre los jóvenes.

No obstante, a pesar de este descenso, las cifras de sedentarismo en los jóvenes evaluados siguen siendo preocupantes, y más aún cuando se contrastan con la prevalencia en personas entre 18 y 64 años en Colombia, reportada en $46.5 \%$ por la Encuesta Nacional de la Situación Nutricional- ENSIN ${ }^{27}$. Esto señala la necesidad de intensificar en la institución educativa las acciones, programas y políticas que promuevan la 
realización de actividad física, en consonancia con la tendencia mundial y nacional.

Lo anterior debe considerar además, las diferencias por género encontradas en este estudio, que coinciden con los reportes realizados por la mayoría de estudios en el tema, así como con los datos de la ENSIN 201027,28. De esta manera, el sedentarismo es más alto en el grupo de mujeres, presentando diferencias significativas en relación con los hombres. Además de las explicaciones sobre las desigualdades en los roles de género ampliamente discutidas por otros estudios, estas diferencias podrían explicarse también a partir del menor tiempo libre para hacer actividad física que reportan las mujeres, debido a la multiplicidad de actividades que deben realizar, incluso desde la juventud, así como a diferencias importantes en las motivaciones que tienen para hacer actividad física ${ }^{8}$.

Otra de las dimensiones poco saludables identificadas en este estudio, fue el manejo del estrés, resultado que coincide con los planteamientos de varias investigaciones en contextos universitarios, que señalan que aspectos como las dificultades o fracasos académicos, alta exigencia de tiempo por parte de los programas académicos, inestabilidad económica, problemas en el funcionamiento familiar, falta de tiempo para el descanso, aspectos relacionados con el grupo social y el apoyo de amigos, los cuales deben ser enfrentados por los jóvenes, generan altos niveles de estrés, e incluso consecuencias en la salud y la percepción de bienestar ${ }^{29}$.

Al igual que en la actividad física, los niveles de estrés fueron más altos en las mujeres, lo cual incrementa su riesgo, considerando que éstas son además, las más sedentarias. Los estudios han explicado la diferencia por género a partir de aspectos psicosociales y biológicos, pero estas diferencias son particularmente importantes de considerar a la hora de diseñar y focalizar intervenciones que mejoren las estrategias para hacerle frente al estrés ${ }^{29}$. Si bien no existen mediciones previas de esta variable en la institución, este sería un aspecto a considerar como prioritario, considerando su impacto, tanto a nivel académico, como personal y social para los jóvenes.

Por otro lado, aunque los resultados muestran que las mujeres son más sedentarias y se estresan más, éstas tienen un menor consumo de sustancias psicoactivas que los hombres, lo cual se convierte en un factor protector frente a diferentes problemáticas. Este resultado coincide por lo informado en otro estudio con universitarios de varias ciudades de Colombia9. Esto podría entenderse a partir de los estereotipos de género y por la presión que ejercen los pares, los cuales terminan reforzando este tipo de comportamientos, de manera más fuerte en los hombres que en las mujeres.

Otro resultado que puede considerarse importante, es el hecho de que los jóvenes que se dedican a estudiar y no trabajan simultáneamente, tienen prácticas más saludables de tiempo de ocio. Este resultado es esperado, considerando que estos jóvenes tienen menos ocupaciones, lo cual les permitiría realizar actividades de disfrute y descanso con mayor frecuencia que quienes además de estudiar, deben trabajar.

Considerando que la realización de actividades que conllevan la búsqueda de satisfacciones, el placer, la inclusión social, la recreación, la ampliación de los intereses culturales, etc. durante el tiempo de ocio permite la satisfacción de necesidades personales, la creación y el desarrollo del sujeto, sería importante pensar dentro las estrategias institucionales que buscan promover el desarrollo integral y el bienestar, el fomento de habilidades para el manejo del tiempo y el apoyo para identificar intereses que les permita a los jóvenes hacer una mejor distribución de su tiempo, tanto en periodos de estudio, como en los de vacaciones ${ }^{30}$. Lo anterior teniendo en cuenta que el ocio y el uso del tiempo libre se consideran en estrecha relación con el poder adquisitivo, el cual condiciona en gran medida el acceso y la elección del tipo de actividades a realizar, pero que podría orientarse hacia la búsqueda de actividades sin costo o de 
acceso libre que pueden promover igualmente, el desarrollo y bienestar de los jóvenes ${ }^{31}$.

Por último, para responder a la intención de observar cómo los hábitos del estilo de vida de estos jóvenes se agrupan entre sí e identificar grupos con mayor riesgo, los resultados del estudio señalaron tres tipologías. La primera de ellas agrupa a los hombres que no trabajan, con prácticas no saludables en actividad física; la segunda a las mujeres que no trabajan, con prácticas no saludables de actividad física y alto nivel de estrés; y la tercera, a las mujeres que no trabajan, con prácticas no saludables de actividad física.

Estas tres tipologías refuerzan el señalamiento sobre la necesidad de promover la actividad física en general en toda la población universitaria de la institución, pero un abordaje diferencial en mujeres alrededor del manejo del estrés. La agrupación de prácticas poco saludables de actividad física (o sedentarismo) en estudiantes que no trabajan $\mathrm{y}$ adicionalmente no tienen pareja, podría poner de relieve que éstos, aun teniendo más tiempo libre que aquellos que trabajan y estudian, no realizan actividad física como parte de sus actividades de ocio. Este aspecto no obstante, debe explorarse con mayor profundidad, para comprender la relación de estas variables.

Con respecto al grupo de mujeres sedentarias y con alto estrés, sería importante considerar acciones focalizadas que les permitan percibir el riesgo potencial de este estilo de vida para su salud, y acompañar cambios que involucren estas dos dimensiones. Por ejemplo, promover la identificación de actividades de su agrado, ya sean estructuradas o cotidianas, que promuevan el movimiento corporal y que a su vez les permitan relajarse y divertirse para controlar el estrés, mejorando su percepción de bienestar y su calidad de vida.

En conclusión, este estudio señala a la actividad física y al estrés como las dimensiones menos saludables de los jóvenes universitarios evaluados, y muestra la agrupación de estas dos dimensiones de manera más fuerte en las mujeres. El tiempo de ocio también se identifica como un aspecto a considerar a la hora de promover los estilos de vida saludables en esta población, particularmente en quienes trabajan mientras cursan sus estudios. Los resultados señalan algunas nuevas preguntas en el tema, que ameritarían la conducción de estudios cualitativos que profundicen en estos aspectos de los estilos de vida de los universitarios, y que podrían llevarse a cabo en los grupos de riesgo identificados.

Con respecto a los resultados de este estudio, cabe señalar como limitaciones metodológicas, la falta de un procedimiento de aleatorización de los estudiantes que fueron incluidos en la muestra, esto debido a que la selección de la muestra respondió más a un muestreo por conveniencia y de sujetos voluntarios, sin embargo, el hecho de contar con una cantidad mayor a los 200 sujetos, permitió utilizar algunos procedimientos inferenciales (obtener valores p por ejemplo), soportando los resultados en la teoría asintótica de la estadística.

\section{Agradecimientos}

Los autores expresan sus sinceros agradecimientos al Centro de Bienestar y al Departamento de Ciencias Sociales de la Pontificia Universidad Javeriana Cali, por la financiación y apoyo de la presente investigación. La participación del segundo autor fue financiada con una beca del programa Jóvenes Investigadores e Innovadores de COLCIENCIAS para el año 2015.

Conflicto de intereses: Ninguno declarado por los autores.

\section{Referencias}

1. Lema L, Varela MT, Duarte C, Bonilla MV. Influencia familiar y social en el consumo de alcohol en jóvenes universitarios. Revista Facultad Nacional de Salud Pública. 2011;29(3):264-271.

2. Salazar I, Varela MT, Lema LF, Tamayo JA, Duarte C, Equipo CEVJU-R Colombia. Evaluación de las conductas de salud en jóvenes universitarios. Revista de Salud Pública. 2010;12(4):599-611. 
3. Organización Mundial de la Salud. Riesgos para la salud de los jóvenes. 2011. Disponible en: http://www.who.int/mediacentre/factsheets/fs345/es

4. Jensen AJ, Ortiz-Salinas ME, Santiago-Huerta Y. Adolescencia y adultez emergente: Un enfoque cultural. Pearson Educación, 2008.

5. Wing M, Russell S, Martin K. Predicting physical activity of first-year university students: an application of the theory of planned behavior. Journal of American College Health. 2009;58(1):45-52.

6. Wiley Al-Nakeeb Y, Lyons M, Dodd L, Al-Nuaim A. An investigation into the lifestyle, health habits and risk factors of young adults. Int. J. Environ. Res. Public Health. 2015;12:4380-4394.

7. Keller S, Maddock JE, Hannöver W, Thyrian JR, Basler HD. Multiple health risk behaviors in German first year university students. Preventive Medicine. 2008;46:189195.

8. Varela, MT, Duarte C, Salazar IC, Lema LF, Tamayo JA. Equipo CEVJU-R Colombia. Actividad física y sedentarismo en jóvenes universitarios de Colombia: Prácticas, motivos y recursos para realizarlas. Revista Colombia Médica. 2011;42(3):269-277.

9. Duarte C, Varela MT, Salazar IC, Lema LF, Tamayo JA. Motivaciones y recursos para el consumo de sustancias psicoactivas en universitarios. Revista Hacia la Promoción de la Salud. 2012;17(1):92-104.

10. Alonso LM, Pérez MA, Alcalá G, Lubo A, Consuegra A. Comportamientos de riesgo para la salud en estudiantes colombianos recién ingresados a una universidad privada en Barranquilla (Colombia). Salud Uninorte. 2008;24(2):235-247.

11. Escobar MP, Pico ME. Autocuidado de la salud en jóvenes universitarios, Manizales, 2010-2011. Revista Facultad Nacional de Salud Pública. 2013;31(2):178186.

12. Arguello M, Bautista Y, Carvajal J, De Castro K, Díaz D, Escobar M, et al. Estilos de vida en estudiantes del área de la salud de Bucaramanga. Revista de Psicología. 2009;1(2):0-0.

13. Quintiliani L, Allen J, Marino M, Kelly-Weeder S, Li Y. Multiple health behavior clusters among female college students. Patient Education and Counseling. 2010;79: 134-137.

14. Spengler S, Mess F, Schmocker E, Woll A. Longitudinal associations of health-related behavior patterns in adolescence with change of weight status and self-rated health over a period of 6 years: results of the MoMo longitudinal study. BMC Pediatrics. 2014;14:242-253.

15. Dood LJ, Al-Nakeeb Y, Nevill A, Forshaw MJ. Lifestyle risk factors of students: A cluster analytical approach. Preventive Medicine. 2010;51:73-77.

16. Feng X, Girosi F, McRae IS. People with multiple unhealthy lifestyles are less likely to consult primary healthcare. BMC Family Practice. 2014;15:126-133.

17. Schnettler B, Peña JP, Mora M, Miranda H, Sepúlveda J, Denegri M, Lobos G. Estilos de vida en relación a la alimentación y hábitos alimentarios dentro y fuera del hogar en la Región Metropolitana de Santiago, Chile. NutrHosp. 2013;28(3):1266-1273.
18. Pons X, Gil M. Patrones de comportamiento, relacionados con la salud en una muestra española de población general. ActaColombiana de Psicología. 2008; 11(1):97-106.

19. Morales M, Ruso C, Llopis A. Comparative study of lifestyle: eating habits, sedentary lifestyle and anthropometric development in Spanish 5- to 15-yrolds. Iran J Public Health. 2015;44(4):486-494.

20. Bel-Serrat S, Mouratidou T, Santaliestra AM, Iacoviello L, Kourides YA, Marild S, Molna D, Reisch L, Siani A, Stomfai S, Vanaelst B, Veidebaum T, Pigeot I, Ahrens W, Krogh V, Moreno LA. Clustering of multiple lifestyle behaviours and its association to cardiovascular risk factors in children: the IDEFICS study. European Journal of Clinical Nutrition. 2013;67:848-854.

21. Moy FM, Hoe VCW, Hairi NN, Buckley B, Wark PA, Koh D, Bueno-de-Mesquita HB, Bulgiba AM. Cohort study on clustering of lifestyle risk factors and understanding its association with stress on health and wellbeing among school teachers in Malaysia (CLUSTer) - a study protocol. BMC Public Health. 2014;14:611-620.

22. Agresti A. An introduction to categorical data analysis. New York: Wiley. 1996; 135 p.

23. Dobson AJ, Barnett A. An introduction to generalized linear models. CRC press; 2008.

24. Hosmer Jr, David W, Stanley L. Applied logistic regression. John Wiley \& Sons; 2004.

25. Ludovic L, Morineau A, Piron M. Statistique exploratoire multidimensionnelle. Dunod, Paris; 1995.

26. Pardo CE, Del Campo PC. "Combinación de métodos factoriales y de análisis de conglomerados en R: el paquete FactoClass." Revista Colombiana de Estadística. 2007;30(2):231-245.

27. Colombia. Instituto Colombiano de Bienestar Familiar. 2005. Encuesta Nacional de la Situación Nutricional en Colombia 2010. Disponible en: http://www.javeriana.edu.co/documents/245769/302 5871/Resumen_Ejecutivo_ENSIN_2010.pdf/160e9856006d-4a60-9da3-d71606703609

28. González S, Sarmiento OL, Lozano O, Ramírez A, Grijalba C. Niveles de actividad física de la población colombiana:desigualdades por sexo y condición socioeconómica. Biomédica. 2014;34:447-459.

29. Arrieta K, Díaz S, González F. Síntomas de depresión, ansiedad y estrés en estudiantes de odontología: prevalencia y factores relacionados. Revista Colombiana de Psiquiatría. 2013;42(2):173-181.

30. Cabrera VE, Salazar PA, Docal MC, Aya VL, Ardila MM, Rivera R. Estilo de vida de los jóvenes y las necesidades de educación sexual. Bogotá: Procuraduría General de la Nación - Instituto de Estudios del Ministerio Público. $2013 . \quad$ Disponible en: http://www.mineducacion.gov.co/cvn/1665/articles340716_archivo_pdf.pdf

31. Salvador T. Adolescentes y jóvenes: ocio y uso del tiempo libre en España. Delegación del gobierno para el Plan Nacional sobre Drogas 2009. Disponible en: http://www.pnsd.msc.es/novedades/pdf/RevisionAdo lescentes.pdf 